\title{
Motion Editing with Data Glove
}

\author{
Wai-Chun Lam \\ City University of Hong Kong \\ 83 Tat Chee Ave \\ Kowloon, Hong Kong \\ email:jerrylam@cityu.edu.hk
}

\author{
Feng Zou \\ City University of Hong Kong \\ 83 Tat Chee Ave \\ Kowloon, Hong Kong \\ email:fzou00@cityu.edu.hk
}

\author{
Taku Komura \\ City University of Hong Kong \\ 83 Tat Chee Ave \\ Kowloon, Hong Kong \\ email:taku@ieee.org
}

\begin{abstract}
In this paper, we propose a new method to edit captured human motion data by using the data glove. The animator first wears a glove and mimics the human body motion observed in the graphical display using his/her hand. Then, a mapping function that converts the motion of the hand to that of the whole body will be generated. Finally, by moving the hand in a slightly different way, a new motion with different taste will be generated. For example, after mimicking the walking motion by alternatively moving the index finger and the middle finger, by quickly moving the fingers with larger strides, it is possible to obtain a running motion. To achieve this goal, a method to map the hand motion to the whole body is proposed. Our method can be used not only for editing human motion, but also for controlling human figures in real time environments such as games and virtual reality systems.
\end{abstract}

\section{Categories and Subject Descriptors}

1.3.7 [ComputerGraphics]: Three-Dimensional Graphics and Realism-Animation

\section{Keywords}

data glove, motion synthesis, motion capture, motion editing, computer puppetry

\section{INTRODUCTION}

Motion editing is often used these days to generate new human motion out of the existing motion captured data in database. To apply the motion to characters with different body size, methods such as retargeting [5] or hierarchical methods [9] have been used. To overlap and blend different motions, methods such as motion warping [14] has been used. In most of the cases, these methods are based on editing key-frame postures; the user needs to determine at which moment the posture must be edited and then change

Permission to make digital or hard copies of all or part of this work for personal or classroom use is granted without fee provided that copies are not made or distributed for profit or commercial advantage and that copies bear this notice and the full citation on the first page. To copy otherwise, to republish, to post on servers or to redistribute to lists, requires prior specific permission and/or a fee.

ACE0́4, June 3-5, 2004, Singapore

Copyright 2004 ACM 1-58113-882-2/04/0006 5.00 . the posture by methods such as inverse kinematics. Finally, the new motion will be generated by blending these keyframes. Although this method is simple and intuitive, there are some drawbacks; first of all, the user cannot find out how the edited keyframe will affect the whole motion until it is replayed; second, in order to drastically edit the motion, a number of keyframes must be inserted. The animators must have enough experience in order to generate motions that satisfy the requirements. These difficulties are due to the gap of the nature of editing methods and that of human motion. Human motion is composed of dynamic variables which change their values time after time. On the other hand, the keyframe-based editing approaches are static editing methods which require the animator to stop the motion at the keyframes and edit the postures in a static manner.

In this paper, we address a new dynamic editing method using a data glove. The ability of human fingers on simulating the motion of legs leads us to this novel solution. As shown in the performance of finger puppet [2], human fingers can simulate human walking, running, and even dancing. First, it is necessary to prepare the real human motion data that is to be used as a basic motion. Then, the animator wears the data glove, display the human motion on the graphical display, and mimics the motion using his/her hand. Taking the general walking motion for instance, the animator needs to move the index finger and the middle finger alternatively synchronous to the human gait appearing on the screen. Then, the mapping function of the motion by the fingers and the body will is created. Using this mapping function, new motions such as running, walking along different paths, or those with larger rotation of the body can be generated. The animator only needs to intuitively change the motion of his/her hand to generate such motion. The motion can be generated in real time; as a result, our method can be used not only for editing human motion, but also for controlling human figures in real time environments such as games and virtual reality systems.

\section{PREVIOUS WORK}

A variety of techniques has been proposed to edit motions of articulated characters; those include interpolation of examples, constrained optimization, and development of task-specific control systems. Interpolation or blending can be used when many motion examples are available $[7,10]$. Such methods require a motion database which may not be available for many animators. Spacetime constraints $[3,4$, $5,6,11]$ is considered as the most effective method to edit 
human motion while preserving the features of the original motion. Although it has been proven that the computational time can be minimized [3], the load still increases proportional to the product of the duration of the motion and the degrees of freedom of the body, it cannot be applied to long-term motions. Therefore, there are still some remaining problems to use spacetime constraints as a tool for interactive motion editing. An interesting approach to interactively generate motion is the approach by Laszlo et al. [8], which directly involves the animator in the control of interactive physics-based simulations of walking, jumping, and climbing using a mix of continuous (mouse-based) and discrete (keypress-based) control. The high level control by the user enables intuitive control, , however, because of the low degrees of freedom of the input devices, intimate editing, which is required in the field of motion editing, could not be achieved. The research done in computer puppetry $[12,13]$ which transforms the performerfs hand motion to the motion of the characters in real-time, have proven the ability of the hand to generate lively motion. Most of the studies in computer puppetry are mainly targeted for facial expressions [13]. Our method focuses on mapping the finger movements to the actions by the whole body.

\section{OUTLINE OF THE METHOD}

As explained in the previous sections, the user first wears the data glove and mimics the human motion with his/her hand including the wrist and the fingers, and then generate the new motions by changing the motion by the hand.

\subsection{Data glove}

This is the key device used in our research to edit the human motion. The data glove used is the P5 glove (Figure 1) by Essentialreality co., Ltd [1]. The position and orientation of the wrist is detected by the infra-red sensors. The flexion/extension of each finger is detected by the bend sensor. The glove uses a 6 bit A/D converter, such that it has a resolution of 64 intermediate positions between a flat and a fisted hand. Although the information that can be captured is quite rough, and the glove cannot capture the adduction/abduction of the fingers, we found the information provided by this glove is enough to achieve our goals in this research.

\subsection{Procedure}

The flow chart of the procedure to edit the motion data is shown in Figure 2. The method we propose in this research can be divided into the capturing stage and the reproduction stage. The procedure done in each stage is as follows:

In the capturing stage, the mapping function that defines the relationship between the motion of the fingers and the human body is generated. At first, the animator mimics the human motion appearing on the graphical display using the hand, as shown in Figure 3. The motion of the index finger and the middle finger are considered to be corresponding to the motion of the legs and the shoulders. Parameters such as the cycle of the motion, the minimum and maximum output value from the sensors will be obtained at this stage. The corresponding parameters of the human motion such as the duration of the motion, and range of the generalized coordinates will also be obtained. To compare the motion of the fingers and the body, we wipe off the high frequency data using Fourier series expansion first. The synchroniza-

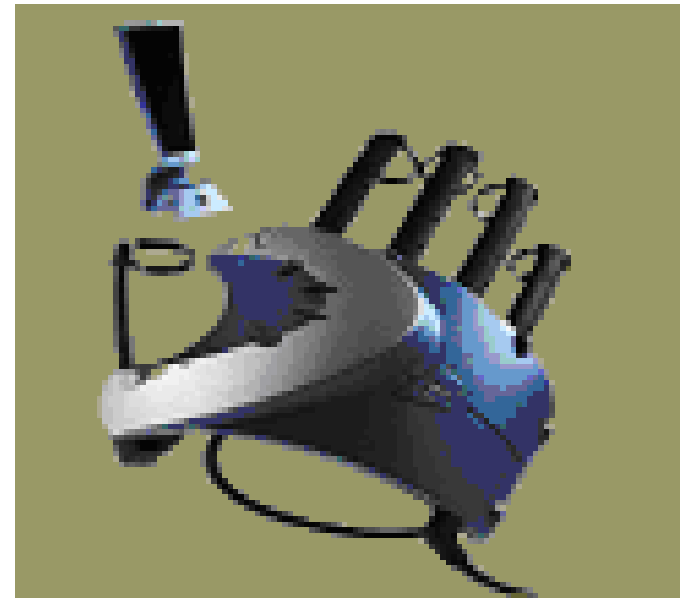

Figure 1: P5 Glove

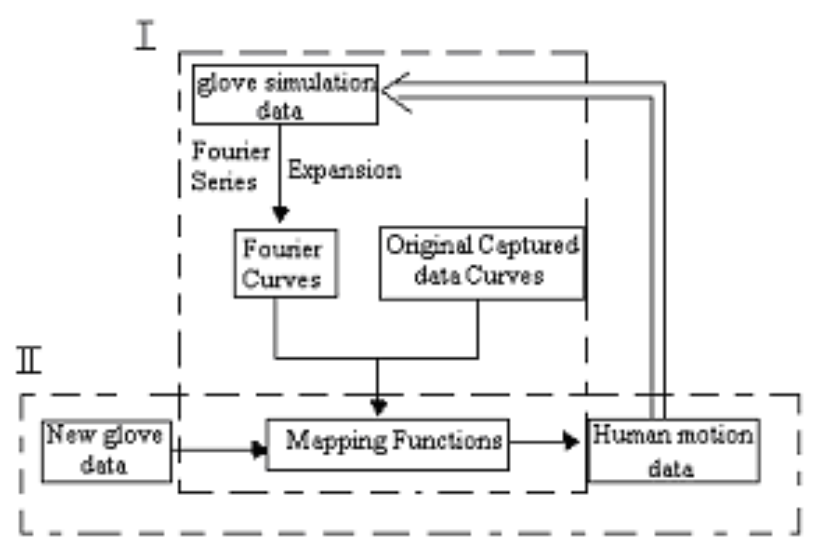

Figure 2: Flowchart of the procedure 


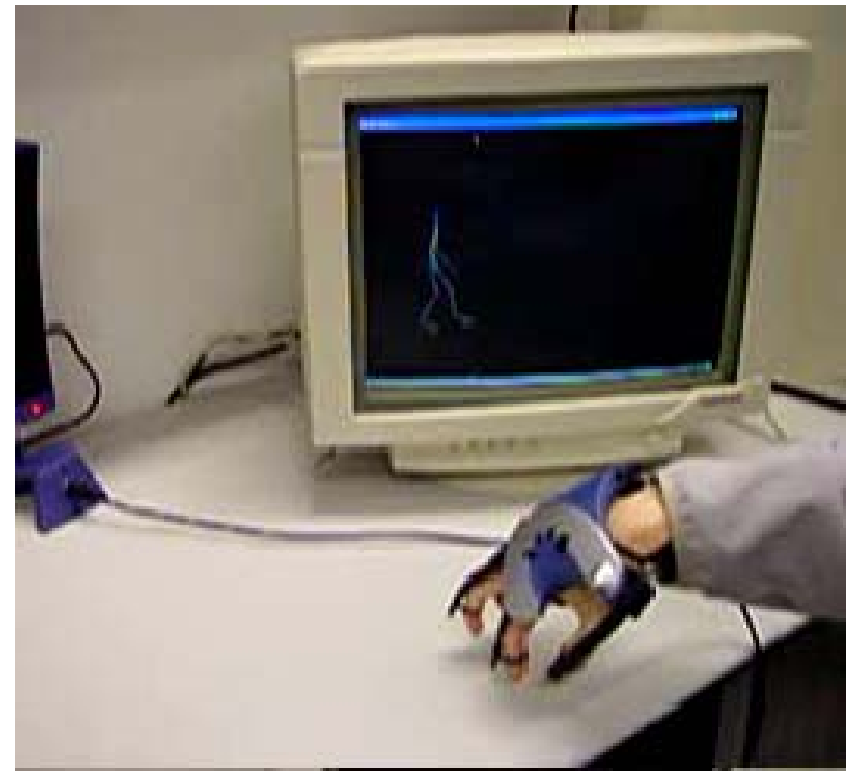

Figure 3: Mimicking the motion appearing on the graphical display by the hand

tion of the two motions is done by matching the timing of the tips and pits of the motion curves. Finally, using this information, the mapping function that converts the hand motion to the human body motion is constructed.

In the reproduction stage, the animator performs a new motion by the hand. The user can change the trajectory of the fingers, and the motion of the wrist to reproduce similar but different motions. For example, if the original clip is a walking motion, it is possible to generate slower gait with larger strides, by changing the corresponding motion by the fingers.

The capturing stage can be repeated several times, if the animator finds that the result is unsatisfactory, because the effectiveness of our method largely relies on the mapping function. The method to generate the mapping function is explained in the following section.

\section{CONSTRUCTION OF MAPPING FUNC- TION}

In this section, the method to map the motion of the hand to the motion of the whole body is explained. In order to do this, first, it is necessary to decide the motion of which finger shall be mapped to which part of the body. Then, it is necessary to decide how the joint angles of the fingers shall be converted to the joint angles of the whole body. The techniques used in this study are explained in the following subsections.

\subsection{Topological matching of the human body and the fingers}

The topology of the human body and the fingers are different. The total degrees of freedom of the whole body are much larger than that of the fingers. Therefore, in order to control the human body model using the fingers, it is necessary to determine which finger motion matches to which generalized coordinate of the whole body. In this research,

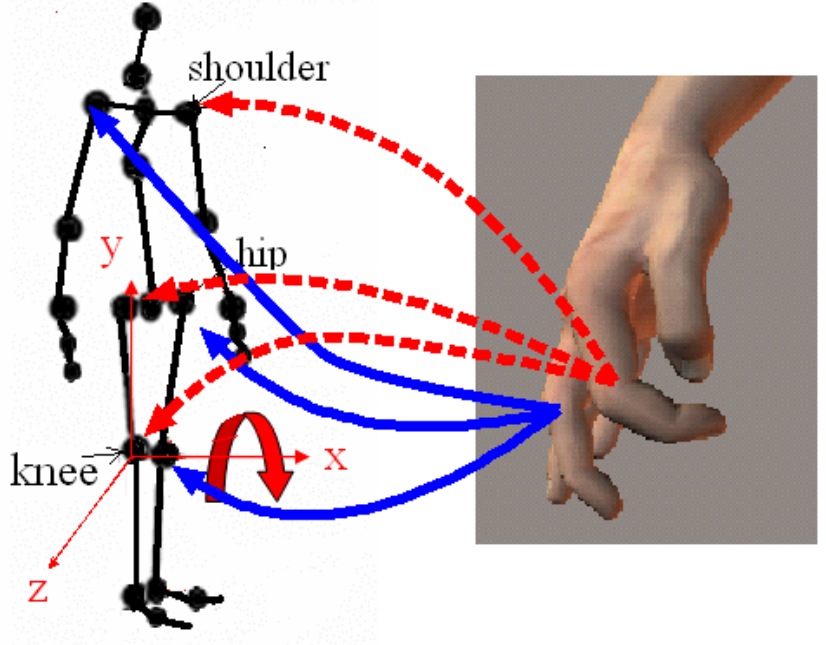

Figure 4: The correlation of finger and the joints of the body used for the mapping. The motion of the index finger is mapped to the left hip, left knee, and right shoulder, and the motion of the middle finger is mapped to the right hip, right knee and left shoulder.

this matching is strictly fixed in advance. This is because when anybody mimic the human motion by their fingers, it is very likely that they mimic the motion of the legs by the index and middle finger. Therefore, we adopted this most natural and intuitive rule to control the body. The joint angles of the hip and knee joint are calculated independently using the flexion of the fingers. Another well known fact with the human motion is that when people control their bodies, the arms are controlled asymmetrically with the legs. For example, when human walk, they move their arm left arm backward when the right leg is swung to the front. Therefore, we have matched the motion by the index finger to that by the right shoulder and the middle finger to that by the left shoulder, as well. These rules of matching are shown in Figure 4. The abduction/adduction and rotation of the thigh and shoulders are not affected by the data glove. This is due to the limitation of the P5 data glove that it cannot capture the corresponding motion of the fingers. This problem, however, is actually not a serious problem, as our method can be easily extended to such motions if a high grade glove that can obtain such data is used.

\subsection{Matching the trajectories}

The matching of the trajectories is done using the tips and pins of the curves. After applying a average filter to the motion data, the time that the tangent of the curve becomes zero are searched in the curves. The period between two pins will be used as the cycle to be matched. Because of the noise and rough A/D conversion, the periodical characteristics of all the trajectories by the glove are unstable and less reproducible. Even though the animator tries to mimic the motion of the body accurately as much as possible, sometimes unintentional problematic motions will be included in the data. Such subtle motions cause serious mal effects when mapping the motions. In order to reduce such effects, a Fourier series expansion is applied to both data to 


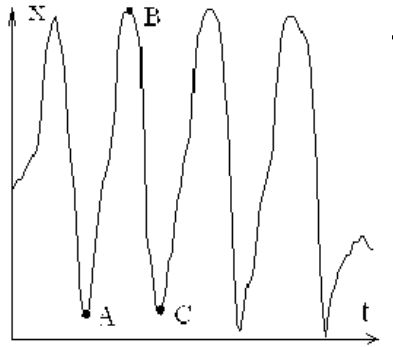

(a)

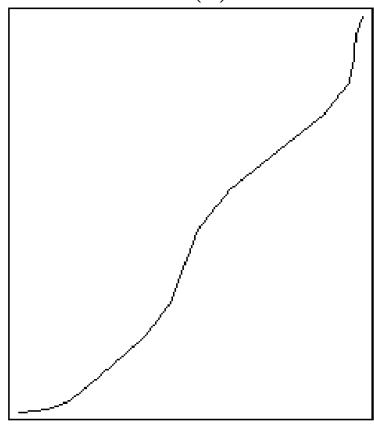

(c)

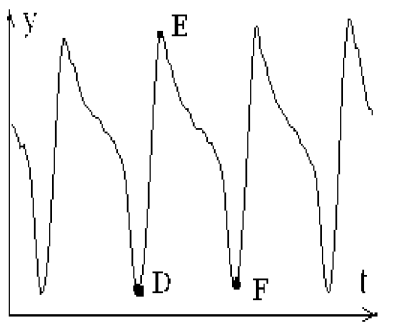

(b)

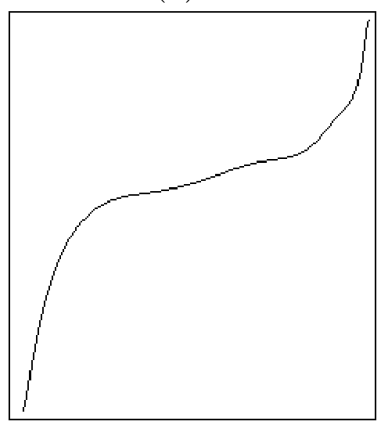

(d)
Figure 5: (a). The trajectory of the finger motion, (b). the corresponding motion by the knee, (c). the mapping funtion between the finger motion and the knee motion in the incremental part, and (d) in the decremental part

remove the noise and problematic high frequency motions from the data.

\subsection{Mapping functions}

Now we have satisfactory curves of the original human lower body and that of the hand. We generate a mapping function for each period between the tip and the pit. This approach is needed as the trajectory of the joint angles of the legs tend to be different when the joints are flexed and extended, where the profile of the fingers tend to be symmetric as shown in Figure 5(a) and 5(b). Multiple points of the trajectory of the hand motion, which are defined here by $x_{i}$, and the corresponding sample points of the hand motion, defined here by $y_{i}$ will be obtained from the motion. Then, the a $2 \mathrm{D}$ curve that interpolates the sampled points $\left(x_{i}, y_{i}\right)$ will be generated using B-splines. This curve is used as the mapping function when the animator performs a new finger motion. Examples of such curves that represent the mapping of the finger and the knee joint during walking are shown in Figure 5. The trajectory of the finger joint shown in Figure 5.a is mapped to the trajectory of the knee joint shown in Figure 5b. Two mapping functions are generated; one for the incremental period between A and B in Figure $5 \mathrm{a}$ and corresponding period between $\mathrm{D}$ and $\mathrm{E}$ in Figure 5b; another is the mapping function that maps the trajectory between point $\mathrm{B}$ and $\mathrm{C}$ in Figure $5 \mathrm{a}$ and the corresponding trajectory between point $\mathrm{E}$ and $\mathrm{F}$ in Figure 5b. Each of them is shown in Figure 5c and 5d, respectively.

\section{MOTION REPRODUCTION}

When a new motion is to be generated by the anima-

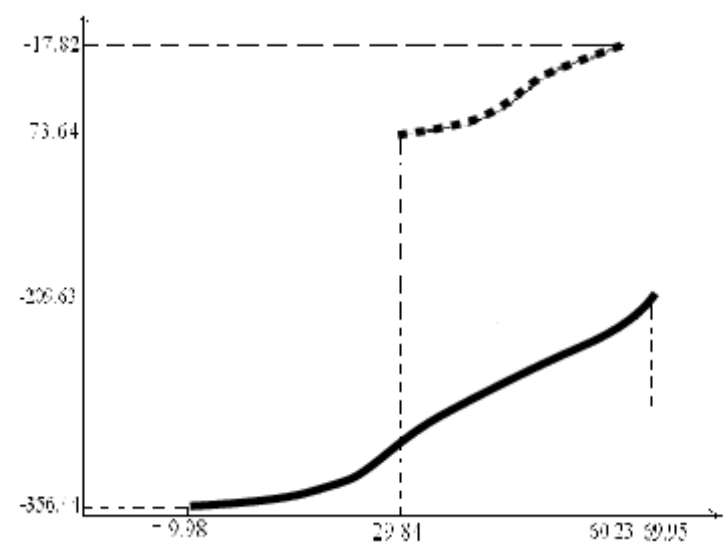

Figure 6: The original mapping function (solid line) and the newly scaled one for editing the motion (dashed line)

tor using the data glove, his/her movements of the fingers must be mapped to the motion of the whole body using the mapping function generated in the previous stage. However, since the range of the values for the new joint angle curves is not the same as the range of the independent variables in the mapping function, it is necessary to deal with those values that are out of the domain. In that case, we scale and transform the domain of the mapping function to that of the range of the new curve. Suppose the domain of the original mapping function ranged from $x_{0}$ to $x_{1}$ in the finger motion and from $y_{0}$ to $y_{1}$ in some body joint motion. When the new finger motion ranges from $X_{0}$ to $X_{1}$, the new joint angle $x$ will be mapped to the corresponding value $y$ as:

$$
\begin{gathered}
y=f\left(\frac{X_{1}-X_{0}}{x_{1}-x_{0}} x\right)\left(\frac{X_{1}-X_{0}}{x_{1}-x_{0}}\right) \\
-\left(x_{0}\left(\frac{X_{1}-X_{0}}{x_{1}-x_{0}}\right)-X_{0}\right)\left(\frac{y_{1}-y_{0}}{x_{1}-x_{0}}\right)
\end{gathered}
$$

where $f()$ is the original mapping function. In Figure 6, an example of the original mapping function (solid line) and the rescaled mapping function (dashed-line) is shown. The upper limit and lower limit of the joint angles are recalculated using Equation (1) as well.

\section{EXPERIMENTS}

An ordinary walking motion captured using a motion capture device was used as the original motion in this experiment. Using this motion, a (1) hopping with large strides and (2) a running motion along a zigzag path was generated. The trajectory of the original gait motion is shown in Figure 7. The animator was asked to mimic the ordinary gait motion using the hand. Through this process, the mapping function was generated. Next, the animator was asked to controlled the hand in a way mimicking a hopping motion. The hand bounced over a desk and the fingers were widely spread when the write moved in the air (Figure 8). The trajectories of the resulting hopping motion are shown in Figure 9. The range of the motion by hip, knee and shoulder joints are all extended, and the motion looks similar to the real running motion. Next, the animator was asked to 


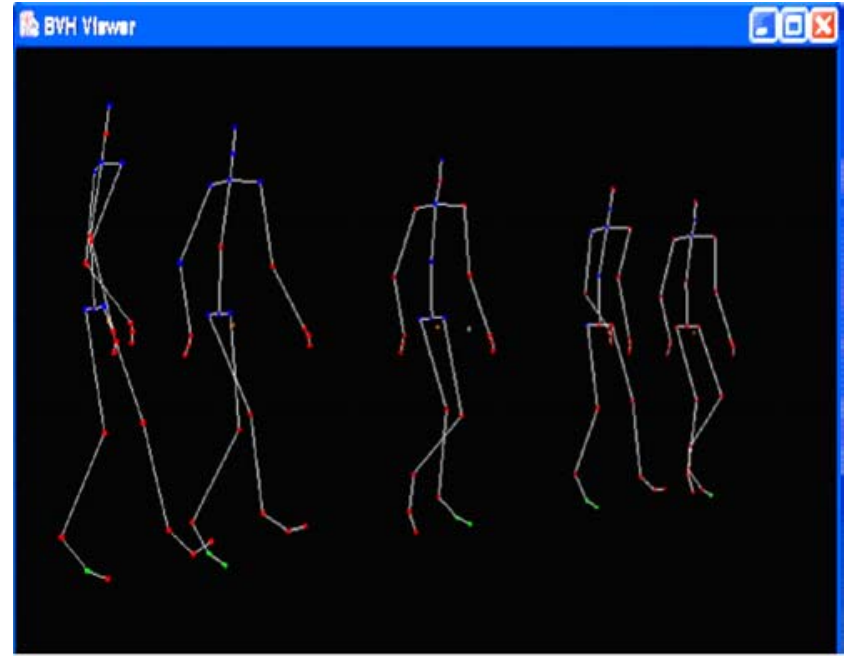

Figure 7: The trajectory of the original gait motion
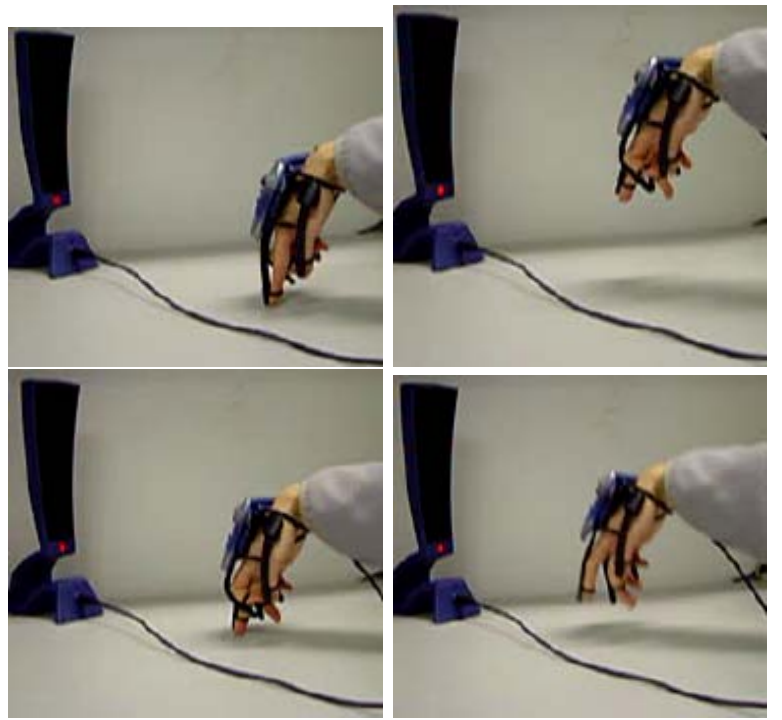

Figure 8: The hand gesture to generate the hopping motion

control the hand along a zigzag path (Figure 10). The final motions are shown in Figure 11.

\section{DISCUSSIONS}

In the zigzag gait, the human model suddenly changes its direction without any tilting of the body, and this phenomenon makes the motion look quite unnatural. This is because when the animator performs the zigzag gait using his/her hand, there is actually no need to tilt the hand to change the direction of the body. Such gap is due to the difference in the dynamics of the motions; the wrist is fully controlled by the arm while the human body utilizes the gravity and the ground reaction force to move around. In order to add such effects, it is necessary to include dynamical effects into the mapping function. The limitation of our method is that the mapping function works properly only when the newly generated motion is similar to the original
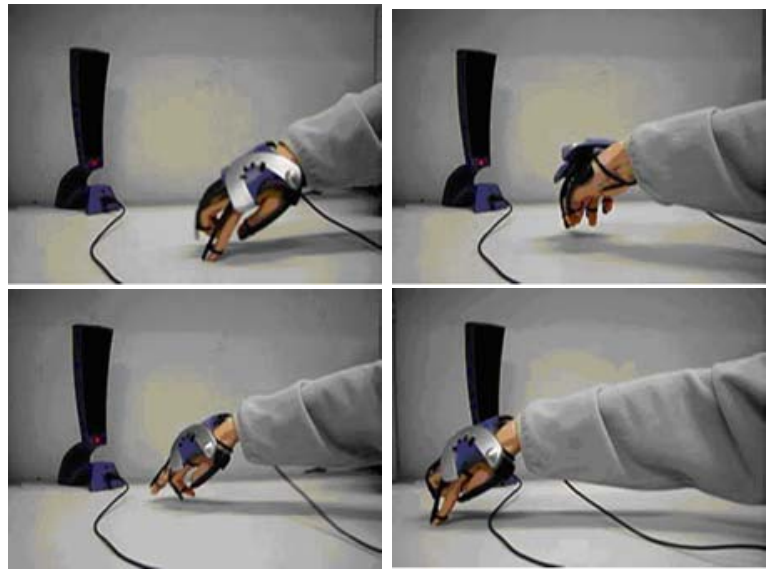

Figure 9: The hand gesture to generate the zigzag walking motion

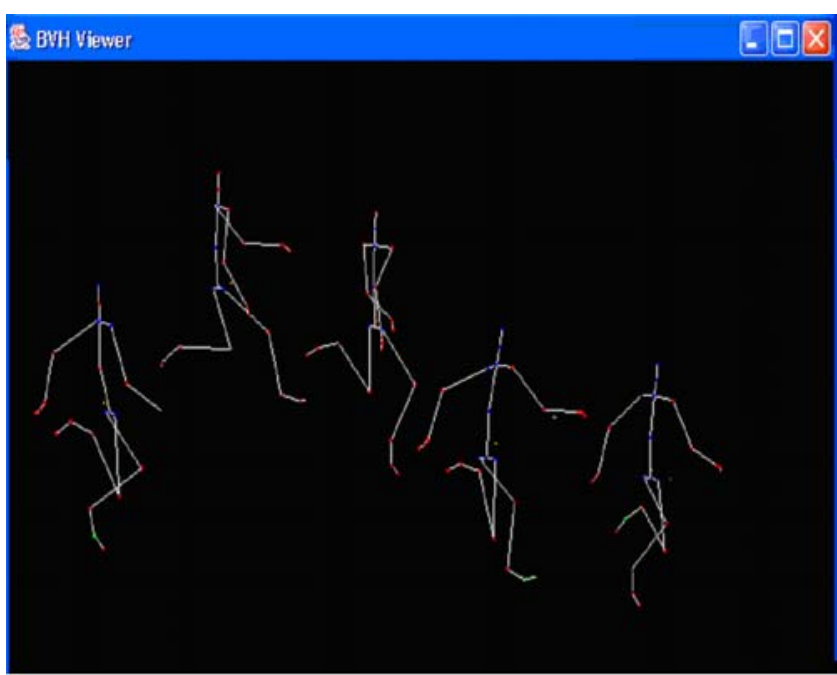

Figure 10: The hopping motion generated by the system

motion. For example, if the original human motion data is a gait motion, the newly generated motion must have a similar topological structure to walking, such as running or hopping. The system does not work correctly if the motion is a totally different one, such as jumping with both legs. Another drawback is that kinematical constraints such as the contact of the feet with the ground are not preserved in the newly generated motion. Therefore, the support foot often slides over the ground which makes the results look unrealistic. In order to improve this, it is necessary to automatically extract such constraints from the original motion, and then keep them preserved in the newly generated motion. The corresponding constraints must be extracted from the fingers too; for example, in case of the constraints between the feet and the ground, it is necessary to find out when the position of the finger tips are in contact with the desk. In that case, a data glove that can give more precise data will be needed. Even though there are many problems to be overcome, the results obtained shows that the hand has a high ability to control human characters; even though the 


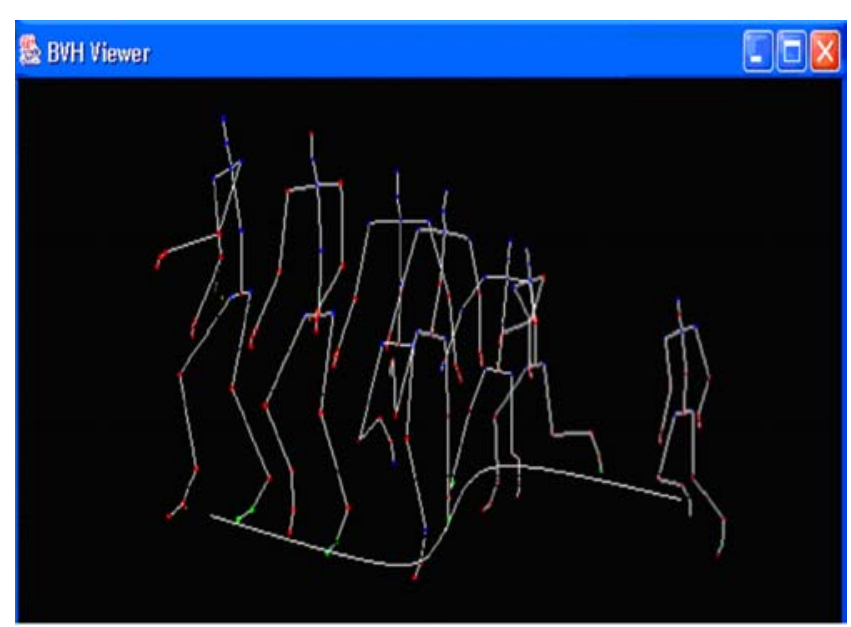

Figure 11: The zigzag walking motion generated by the system

input from the glove is rough, we could still generate vivid motion by the characters in a simple and intuitive way. By overcoming the problems discussed above, it is possible to consider our approach can be used as a new method to edit human motion data.

\section{CONCLUSION AND FUTURE WORK}

In this paper, we proposed a new method to edit human motion using the data glove. Using the input human motion data, it is possible to generate various new motions using the data glove. Even we limited the input to a single motion data, another option to improve the results is to generate the motion not only from the original motion but by the blending of several similar but different motions. For example, if we have motions such as hopping and running in addition to ordinary walking in the database, and if the system can automatically select such motions to be used for the newly generated motion when the animator controls the glove in a way that is similar to such motions, then the results have a chance to look more natural and realistic. The corresponding joints of the fingers and those of the whole body were strictly fixed in this research. Although this approach is enough for the motion we have introduced in this paper, it would be far more convenient for the animators if the best matching joints were automatically found by comparing the basic human motion and the motion of the fingers and the wrist. This can be considered as the next step for this research.

\section{REFERENCES}

[1] http://www.essentialreality.com/p5_glove.asp.

[2] http://www.thepuppetstudio.com/what.html.

[3] A. C. Fang and N. S. Pollard. Efficient synthesis of physically valid human motion. ACM Transactions on Graphics, 22(3):417-426, 2003.

[4] M. Gleicher. Motion editing with spacetime constraints. Proceedings of Interactive 3D Graphics, pages 139-148, 1997.

[5] M. Gleicher. Retargetting motion to new characters. Computer Graphicsi Proceedings, Annual Conference Series, pages 33-42, 1998.
[6] M. Gleicher and P. Litwinowicz. Constraint-based motion adaptation. The Journal of Visualization and Computer Animation, 9:65-94, 1998.

[7] L. Kovar, M. Gleicher, and F. Pighin. Motion graphs. ACM Transactions on Graphics, 21(3):473-482, 2002.

[8] J. Laszlo, M. van de Panne, and E. Fiume. Interactive control for physically-based animation. In proceedings of SIGGRAPH 2000, pages 201-208, 2000.

[9] J. Lee and S. Y. Shin. A hierarhical approach to interactive motion editing for human-like figures. Computer graphics proceedings, annual conference series, pages 39-48, 1999.

[10] Y. Li, T. Wang, and H.-Y. Shum. Motion texture: A two-level statistical model for character motion synthesis. ACM Transactions on Graphics, 21(3):465-472, 2002.

[11] Z. Popović and A. Witkin. Physically based motion transformation. Computer graphics proceedings, annual conference series, pages 11-20, 1999.

[12] H. J. Shin, J. Lee, S. Y. Shin, and M. Gleicher. Computer puppetry: An importance-based approach. ACM Transactions on Graphics, 20(2):67-94, 2001.

[13] D. J. Sturman. Computer puppetry. Computer Graphics in Entertainment, pages 38-45, 1998.

[14] A. Witkin and Z. Popovi', Motion warping. Computer Graphics (Proceedings of SIGGRAPH 95), pages 105-108, 1995. 